\title{
Genetic Characterization of Multi Drug Resistant Enterobacteriaceae
}

\author{
Ranjitha Shankare Gowda ${ }^{1}$, Vijay Kumar Gowdara Shankarappa ${ }^{2}$, Ramya Singipura \\ Ramalingaiah $^{3}$, Krishna Karthik. Manthravadi ${ }^{4 *}$ and Satya Sai Badveti ${ }^{4}$ \\ ${ }^{1}$ Department of Microbiology, ${ }^{4}$ Department of Microbiology, JSS Medical College, JSS \\ University, Mysore, Karnataka, India \\ ${ }^{2}$ Department of Microbiology, Kodagu Institute of Medical Sciences, Madikeri, India \\ ${ }^{3}$ Department of Microbiology, Pondicherry Institute of Medical Sciences, Pondicherry, India
}

*Corresponding author

\section{A B S T R A C T}

\section{Keywords \\ Uropathogenic \\ Enterobacteriaceae, Global \\ public health problems, \\ Molecular methods, \\ Surveillance tools \\ Article Info \\ Accepted: \\ 24 August 2018 \\ Available Online: \\ 10 September 2018}

Molecular methods that enable the detection of antimicrobial resistance determinants are critical surveillance tools that are necessary to aid in curbing the spread of antibiotic resistance. The objectives of the study were to determine the prevalence of multidrug-resistant Enterobacteriaceae species isolated from the urine samples in a tertiary care hospital and to detect their drug resistance patterns and their genetic characterization.

\section{Introduction}

Enterobacteriaceae are Gram-negative rods with a length of $1-3 \mu \mathrm{m}$. They are facultative anaerobes, oxidase-negative, catalase positive, and grow on MacConkey agar, and their natural hosts are human and animal intestines (Forbes et al., 2007). There are 44 genera and approximately 176 species (Brenner et al., 2005). The normal flora include Escherichia coli, Enterobacter spp., Klebsiella spp., Morganella spp., Proteus spp., Providencia spp., and Serratia spp., and the obligate human pathogens include Salmonella spp., Shigella spp. and Yersinia spp (Brooks et al., 2010). Enterobacteriaceae may account for $80 \%$ of clinically significant isolates of Gramnegative bacilli and $50 \%$ of clinically significant bacteria in clinical microbiology (Murray et al., 2003). Some Enterobacteriaceae species have only been identified based on their16S rDNA sequence and have not been isolated or characterized biochemically. Antibiotic resistance is one of the few examples of evolution that can be addressed experimentally. It is widely 
accepted that antibiotics and amp; antibiotic resistance genes play fundamental etiological roles as weapons \&amp; shields in shaping the structures of microbial communities. The expression of antibiotic resistance determinants must therefore be tightly regulated. Understanding resistance thus requires the analysis of regulatory networks controlling bacterial evolvability, the physiological webs affected and the metabolic re-wiring it incurs (Martinez et al., 2009). Members of the family Enterobacteriaceae are the leading nosocomial pathogens causing variety of Hospital acquired infections (HAI), most commonly urinary tract infections (UTI). Escherichia coli (E.coli) is the most common Nosocomial uropathogen. Antibiotic abuse, increased usage of antibiotics in veterinary and agriculture fields has resulted in E. coli becoming multidrug resistant (MDR). Antimicrobial resistance development could be chromosomally mediated, plasmid mediated or due to production of enzymes which will hydrolyze the drug and makes it ineffective. Beta lactamase enzymes like extended spectrum beta lactamases (ESBL's), AmpC beta lactamases and Metal lo-beta lactamases (MBL's) have induced resistance to all beta lactams including carbapenems (Patterson et al., 2009). Detection of these enzymes production by enterobacterial nosocomial agents is essential in correct management of these infections. In view of increasing antimicrobial resistance development in Enterobacteriaceae, it is important that the mechanism of resistance development is also detected.

\section{Materials and Methods}

This study was conducted over the period of 1 year, Patients of all age groups and of both genders either admitted or attending OPD Clinics at a tertiary care hospital, Mysore and Clinically suspected as cases of Urinary tract infections and whose urine culture yielded
Enterobacteriaceae only were included and Patients whose urine culture yielded other bacterial uropathogens were excluded from the study.

\section{Bacterial identification and antimicrobial susceptibility testing results}

Out of the1435 positive urine cultures, Enterobacteriaceae uropathogens were isolated in 743 samples $(51.8 \%)$. Out of 743 Enterobacteriaceae isolates, E. coli $46.30 \%$ was the predominant uropathogen. Klebsiella uropathogen rate was $4.5 \%$ (Table 1). Highest number of UTI due to Enterobacteriaceae were found in patients above 50yrs age $(31.2 \%)$, followed by patients in $4^{\text {th }}$ decade age group (25.84\%). UTI due to uropathogenic Enterobacteriaceae was high in males $(56.8 \%)$ in comparison with females $(43.2 \%)$. Isolation rate of Enterobacteriaceae in CAUTI was $(40.24 \%)$ and $(59.8 \%)$ in HAUTI's (Table 2). Overall HAUTI rate was higher than CAUTI. 391 (52.42\%) out of 743 were enzyme producers. $157(40.15 \%)$ were Community acquired UTI's (CA-UTI) and $234(59.85 \%)$ were nosocomial or hospital acquired UTI's (HA-UTI). Uropathogenic species of Enterobacteriaceae liberate 3 types of enzymes (ESBL, AmpC and Carbapenemase) which make the beta lactam antibiotics ineffective. Some Uropathogens may release two types of enzymes resulting in multi-drug resistance development. Only ESBL production was seen in $304(77.74 \%)$ isolates out of 391. 128 were CA-UTI $(42.10 \%)$ and $176(57.90 \%)$ were nosocomial UTI's.66 isolates were only AmpC producers (16.87\%). 17 (25.75\%) and $49(74.25 \%)$ were CA-UTI and HA-UTI respectively (Table 3 ). Combined ESBL and AmpC production was seen in 12 CA-UTI's and 9 HA-UTI's respectively. $21(5.37 \%)$ isolates were found to have dual enzyme production. Surprisingly $12(57.14 \%)$ of these were isolated from CAUTI and $09(42.86 \%)$ were from HA-UTI. 
Isolates having dual enzyme production exhibit resistance to several drugs including Aminoglycosides, SulphamethaxozoleTrimethoprim and urinary quinolones. Among 304 ESBL producing isolates, 270 (88.81\%) were E.coli, 23 (7.56\%) were Klebsiella species, 09 (2.96\%) were Citrobacter species and only $02(0.65 \%)$ were Proteus isolates. Out of 66 AmpC producers, 57 (86.36\%) were E.coli, 04 (6.06\%) were Klebsiella species, 04 $(6.06 \%)$ were Proteus species and 01(1.51\%) was Citrobacter species. $17(80.95 \%)$ out of the 21 dual enzyme producers were E. coli. Among the 176 hospital acquired ESBL producing strains, 158 were E.coli $(89.77 \%)$, 13 were Klebsiella (7.38\%) and 05 were Citrobacter species (2.84\%). Out of the 49 hospital acquired cases of AmpC, 44 were E.coli (89.79\%). Among the 270 E.coli which were "only ESBL" producers, highest rate of resistance $(95.56 \%)$ to Cephalosporins and Nalidixicacid $(93.7 \%)$ was observed, followed by Norfloxacin $(91.48 \%)$ and Cotrimoxazole $(63.33 \%)$. Imipenem resistance was the lowest (4.81\%). Amikacin, Nitrofurantoin and Piperacillin - tazobactum resistance was seen in $11.8 \%, 16.6 \%$ and $17.8 \%$ respectively. In our study Klebsiella species isolates also showed a very high resistance to 3rd generation cephalosporins (82.6\%). Resistance to Fluroquinolones, and Nalidixicacid was seen in $39.1 \%$ and $30.4 \%$ Klebsiella isolates were resistant to Trimethoprim Sulphamethaxazole. Like the E.coli isolates Klebsiella isolates in the present study showed a low resistance rate to Imipenem (4.35\%), Nitrofurantoin (13\%), Piperacillin-tazobactum (17.3\%) and Amikacin (21.7\%). In contrast to the ESBL producers which showed highest resistance to Cephalosporins, Amp C producing $E$. coli showed highest resistance to Cotrimoxazole (91.22\%) followed by Cephalosporins (87.72\%). Other drugs like Piperacillin-Tazobactam (59.65\%) and Amikacin (43.86\%) showed more resistance when compared to ESBL producers in whom they could be used quite promisingly. Least resistance was to Imipenem and Nitrofurantoin together $(24.56 \%)$. All the 04 Klebsiella isolates producing AmpC enzyme were susceptible to Imipenem and Nitrofurantoin. The resistance pattern of dual enzyme producers was worth noticing. $100 \%$ resistance was seen to Cephalosporins and Nalidixic acid among the E. coli isolates and $100 \%$ resistance to Cephalosporins, Amikacin, Nalidixic acid and Cotrimoxazole among the Klebsiella isolates. With this finding, it can be concluded that the severity of infection and drug resistance would be more when the etiological agent produces both the enzymes ESBL and Amp C.

\section{Genetic characterization}

Randomly chosen isolates which were phenotypically confirmed as ESBL or Amp C producers were subjected for PCR study. PCR study of 40 phenotypically confirmed ESBL E. coli showed CTX-M gene positivity in 33 isolates (82.55), but all of them were negative for SHV gene. Similarly, out of 15 Klebsiella isolates, 11 were positive $(73.33 \%)$ for CTX$\mathrm{M}$ gene and all were negative for SHV gene (Table 4 and 5). Thus majority of our ESBL isolates were CTX-M producers. Interestingly, out of the 20 E. coli and 4 Klebsiella AmpC producers tested for the 2 genes ACC and DHA, (Table 6 and 7) all were negative for both the genes.

\section{Results and Discussion}

UTI is the most common HAI both in developed and developing countries (Chatterjee et al., 2009). Lack of properly equipped microbiology laboratory, nonavailability of an experienced clinical microbiologist, failing to test for the enzyme production and lack of knowledge about these MDR pathogens are the contributory factors for the high UTI rate (Suresh et al., 2009). 
UTI is a global problem in health care setups. The rate of UTI varies from place to place. UTI rate in this one year prospective study was $26.42 \%$. Most of the hospitals have similar rate of HAUTI worldwide. A low UTI rate has been found in those centers adopting good hospital practices. It is well known that HA UTI rate is high globally. In our study also HA UTI rate was very high $(69.69 \%)$, CA UTI rate was $30.31 \%$. The most common agents of HA or CA UTI are Enterobacteriaceae species across the world (Kahlmeter et al., 2003). The results of the study were in concordance with that of several studies. $51.8 \%$ isolates were Enterobacteriaceae and Remaining 48.2\% isolates were Enterococci, Coagulase neative Staphylococci, Candida species, Pseudomonas aeruginosa and Acinetobacter species. Isolation rate was similar to many studies (Kahlmeter et al., 2003). Urinary cathetarization and diabetes mellitus are the most common factors favoring occurrence of UTI in hospitalized patients. In the recent years development of Multi drug resistance in uropathogens is a common observation in all the multi speciality health care centers. Antibiotic resistance in bacteria is manmade. There are several contributory factors. Antibiotic misuse and unnecessary prescription of antibiotics is the major factor, failure to comply with the schedule, poor quality of drugs and over usage of antibiotics in agriculture and veterinary science are also responsible for the development of this serious problem. This is posing a serious problem in the management of nosocomial infections including UTI. With no new molecule production in the pipeline and MDR development to the existing antibiotics among the nosocomial strains has resulted in a grave situation. Fortunately urinary antibiotics like Nitrofurantoin are not misused, hence we still have a safe and effective antibiotic to treat UTI's. Production of Beta lactamase enzymes inactivating the beta lactam antibiotics is the most common antibiotic resistance mechanism acquired by Enterobacteriaceae. By genetic mutation, bacteria acquire the ability to produce this enzyme. Extended beta lactamase, AmpC beta lactamase and carbapenamase are the different beta lactams which play a pivotal role in drug resistance development in uropathogenic Enterobacteriaceae. These strains inhabit the hospital environment and are the leading agents causing HAI's including UTI. These beta lactamase producing Enterbacteriaceae inactivate the beta lactam antibiotics including the $3^{\text {rd }}$ generation Cephalosporins and Monobactams. $52.42 \%$ of the isolates in the present study were Enzyme producing Enterobacteriaceae. Almost $60 \%$ of these were HA UTI agents and $40 \%$ were found in CA UTI cases. This is relatively a high rate of CA UTI. This increased rate of CA UTI caused by enzyme producing uro pathogens could be due to day care treatment practice, patient being in incubation period at the time of discharge. This spillage is the cause for a high CA UTI globally. $78 \%$ of isolates produced only Extended spectrum beta lactamase (ESBL) enzyme, $42 \%$ of these were from CA.UTI and $58 \%$ were isolates from HAUTI. In these isolates high resistance was seen in Cephalosporins and Nalidixic acid followed by Norfloxacin and Cotrimoxazole. ESBL production is an important character acquired by Gram negative bacilli including species of Enterobacteriaceae. Modern diagnostic and therapeutic manoeuvres, forced immunosuppression, unnecessary overstay in hospitals and improper antibiotic usagehas resulted in increase in UTI due to MDR bacteria (Eshwarappa et al., 2009). AmpCbeta lactamase belongs to Bush group 1, Ambler class $\mathrm{C}$ beta lactamase. Action of AmpC enzyme is not affected by beta lactamase inhibitors like Clavulanic acid, Tazobactum or Sulbactum. AmpC production could be chromosomal or plasmid mediated. Chromosomal AmpC is an inducible beta lactamase. 
Table.1 Showing total number of Enterobacteriaceae isolates

\begin{tabular}{|l|c|}
\hline Total number of culture positive samples & 1435 \\
\hline Total number of Enterobacteriaceae isolates & 743 \\
\hline Percent Enterobacteriaceae isolates & $51.8 \%$ \\
\hline
\end{tabular}

Table.2 Showing Enterobacteriaceae isolation rate in hospital and community acquired cases of UTI

\begin{tabular}{|l|l|l|}
\hline Enterobacteriaceae isolates & 743 & \\
\hline Community acquired & 299 & $40.2 \%$ \\
\hline Hospital acquired & 444 & $59.8 \%$ \\
\hline
\end{tabular}

Table.3 Showing enzyme positive and enzyme negative among Enterobacteriaceae isolates

\begin{tabular}{|l|l|l|}
\hline Total Enterobacteriaceae isolated= $\mathbf{7 4 3}$ & Percent \\
\hline Enrayme positive & 391 & $52.6 \%$ \\
\hline Enrayme negative & 352 & $47.4 \%$ \\
\hline
\end{tabular}

Table.4 Showing genetic analysis- PCR result of ESBL E.coli

\begin{tabular}{|l|c|c|c|}
\hline & CTX-M & SHV & CTX-M + SHV \\
\hline Positive & 33 & 0 & 0 \\
\hline Negative & 07 & 40 & 40 \\
\hline Total organisms tested & 40 & 40 & 40 \\
\hline
\end{tabular}

Table.5 Showing genetic analysis- PCR result of ESBL Klebsiella

\begin{tabular}{|l|c|c|c|}
\hline & CTX-M & SHV & CTX-M + SHV \\
\hline Positive & 11 & 0 & 0 \\
\hline Negative & 04 & 15 & 15 \\
\hline Total organisms tested & 15 & 15 & 15 \\
\hline
\end{tabular}

Table.6 Showing genetic analysis- PCR result of AmpC E. coli

\begin{tabular}{|l|c|c|c|}
\hline & ACC & DHA & A CC+DHA \\
\hline Positive & 0 & 0 & 0 \\
\hline Negative & 20 & 20 & 20 \\
\hline Total organisms tested & 20 & 20 & 20 \\
\hline
\end{tabular}


Table.7 Showing genetic analysis- PCR result of AmpC Klebsiella

\begin{tabular}{|l|c|c|c|}
\hline & ACC & DHA & ACC+DHA \\
\hline Positive & 0 & 0 & 0 \\
\hline Negative & 4 & 4 & 04 \\
\hline Total organisms tested & 04 & 04 & 04 \\
\hline
\end{tabular}

Persisting with an ineffective 3rd generation cephalosporin antibiotic in a chronic case of UTI results in increased enzyme production, in turn to increased drug resistance. The plasmid mediated AmpC beta lactamases are resistant to 3rd generation Cephalosporins and Cephamycins but this enzyme do not act on Cefepime.

Action of AmpC is less in comparison with ESBL's. AmpC production was also seen in $86.36 \%$ of E.coli and6.06 \% Klebsiella isolates. In our study AmpC producing E.coli and K.pneumoniae were resistant to Nalidixic acid, Nitrofurantoin, Aminoglycosides, and Sulphadrugs, apart from resistance to Cephalosporins. Resistance to Imipenem was less in these isolates. Some bacteria have evolved more than one mechanism of drug resistance development.

Dual enzyme producing uropathogens are more often isolated from patients with chronic UTI, in patients with congenital abnormality in the renal system and in patients with renal calculi. Prolonged catheterization predisposes to infection with agents producing dual beta lactamase enzymes. These strains showed 100\% resistance to Cephalosporins, Nalidixic acid and Norfloxacin. Imipenem was found to be effective against these strains. 21 isolates out of 391 enzyme producers were dual enzyme producing strains. $17(80 \%)$ were E.coli and $4(20 \%)$ were $K$. pneumoniae strains. Dual enzyme producing uropathogen isolation is reported from studies by Sinha $e t$ al., (2008), Vandana et al., (2009) and Rajini et al., (2008). A well-equipped clinical microbiology laboratory offering detection of enzyme production in uropathogens will be of great help in controlling the rate of UTI in any multi-specialty hospital. From the results of the present study it is understood that UTI is one of the main health problem both in Community and hospital setting. Proper knowledge of detecting and identifying the different uropathogens, their drug susceptibility pattern and their drug resistance mechanism is very vital in the correct management of UTI. E.coli is the most common uropathogen globally. Klebsiella pneumoniae species and others are less common.

Prolonged hospital stay, improper antibiotic prescription and defaulting by the patient results in chronicity and antibiotic resistance development. Antibiotic resistance is mainly due to production of enzymes inactivating the drugs. Screening of all uropathogens for detection of these enzymes helps in choosing the correct antibiotic. An effective antibiotic stewardship and implementation of antibiotic policy would help in controlling the escalation of MDR UTI cases in many hospitals

\section{References}

Agarwal, J. and Singh, M. (2012). Pathogenomics of uropathogenic Escherichia coli. Indian Journal of Medical Microbiology 30(2), 141-9.

Barnett, and David, S. (1997). Global Symposium on Aging: Management of Geriatric Lower Urinary Tract Disorders. Urinary Tract Infection: An Overview. American Journal of the Medical Sciences: Volume 314 Issue 4 - pp 245-249. 
Brenner, D.J. and Schleifer, K.H. (2005). 2nd Edition. Michigan State University, Springer, East Lansing.

Brooks, G.F. and Mietzner, T.A. (2010). Medical Microbiology. Jawetz, Melnick and Adelbergs, 25th Edition, McGraw-Hill Companies, 213-219.

Chatterjee, B. (2009). Antimicrobial resistance stratified by risk factor among Escherichia coli strains isolated from the urinary tract from a rural clinic in central India. Indian Journal of Medical Microbiology 27(4): 329-34

David, L. (2009). Resistance in gram negative bacteria: Enterobacteriaceae. American Journal of Infection Control. S26 Volume 34. Number 5 supplement I

Eshwarappa, M. (2011). Clinic Microbiological profile of urinary tract in South India. Indian Journal of Nephrology, volume 21.

Forbes, B.A. and Bailey, W.R. (2007). Bailey\& Scott's Diagnostic Microbiology. $12^{\text {th }}$ Edition, Elsevier Mosby, St. Louis.

Jose Luis Martinez. (2009). A global view of antibiotic resistance. FEMS Microbiology Reviews, Volume 33, Issue 1, 1 January 2009, Pages 44-65.

Kahlmeter, G. (2003). An international survey of the antimicrobial susceptibility of pathogens from uncomplicated urinary tract infections: the ECO.SENS Project. Medicine Journal of Antimicrobial Chemotherapy Volume 51, Issue 1 Pp. 6976.

Murray, P.R. and Yolken, R.H. (2003). Enterobacteriaceae. Introduction and Identification. In: Farmer, III, J.J., Eds., Manual of Clinical Microbiology, Elsevier, Philadelphia, 647.
Rajini, E. and Anuradha. (2008-01 - 2008-02). Detection of Extended-Spectrum $\beta$ lactamases in AmpC $\beta$-lactamaseProducing Nosocomial Gram-negative Clinical Isolates from a Tertiary Care Hospital in Delhi. Indian Journal for the Practicing Doctor. Vol. 4, No. 6

Savas, L. and Onlen, Y. (2006). Nosocomial urinary tract infections: micro-organisms, antibiotic sensitivities and risk factors. West Indian med. $j$. vol.55 no. 3 Mona

Savitha, T. and Thangamariappan, K. (January, 2011). Prevalence study on emergence of urinary tract infection in Erode, Tamil Nadu, India. International Journal of Current Research, Vol.2, Issue, 1, pp. 067072.

Sinha, P. and Pathak, D. (2008). Prevalence of extended spectrum beta lactamase and AmpC beta lactamase producers among Escherichia coli isolates in a tertiary care hospital in Jaipur. Indian J Pathology and Microbiology. Jul-Sep; 51(3):367-9, 2008

Suresh, J. Antony. The changing epidemiology of Extended spectrum beta lactamase infection of the urinary tract focusing on clinical resistance and therapeutic options. www.intechopen.com

Vandana, K.E. and Honnavar, P. (2009). AmpC Beta Lactamases Among ESBL Producing Escherichia Coli And Klebsiella Pneumoniae. If You Don't Look, You Won't Find. Journal of Clinical and Diagnostic Research. (3): 1653-1656

Vinod Prabhu, V. and Selvaraj Pandian, R. (2012). Study on the prevalence of Urinary tract infection in the Paliyar Indian tribe. International Journal of Current Research. Vol.4, Issue, 01, pp. 044-048.

\section{How to cite this article:}

Ranjitha Shankare Gowda, Vijay Kumar Gowdara Shankarappa, Ramya Singipura Ramalingaiah, Krishna Karthik. Manthravadi and Satya Sai Badveti. 2018. Genetic Characterization of Multi Drug Resistant Enterobacteriaceae. Int.J.Curr.Microbiol.App.Sci. 7(09): 3532-3538. doi: https://doi.org/10.20546/ijcmas.2018.709.438 